\title{
A novel approach in ECG beat recognition using adaptive neural fuzzy filter
}

\author{
Glayol Nazari Golpayegani ${ }^{1}$, Amir Homayoun Jafari ${ }^{1}$ \\ ${ }^{1}$ Biomedical Engineering Department, Islamic Azad University, Science and Research Branch, Tehran, Iran. \\ Email: Gelayol777@yahoo.com,Amir_j73@yahoo.com \\ Received Jan. $5^{\text {th }}, 2009$; revised Jan. $16^{\text {th }}, 2009$; accepted Feb. $10^{\text {th }}, 2009$
}

\begin{abstract}
Accurate and computationally efficient means of electrocardiography (ECG) arrhythmia detection has been the subject of considerable research efforts in recent years. Intelligent computing tools such as artificial neural network (ANN) and fuzzy logic approaches are demonstrated to be competent when applied individually to a variety of problems. Recently, there has been a growing interest in combining both of these approaches, and as a result, adaptive neural fuzzy filters (ANFF) [1] have been evolved. This study presents a comparative study of the classification accuracy of ECG signals using (MLP) with back propagation training algorithm, and a new adaptive neural fuzzy filter architecture (ANFF) for early diagnosis of ECG arrhythmia. ANFF is inherently a feed forward multilayered connectionist network which can learn by itself according to numerical training data or expert knowledge represented by fuzzy if-then rules [1]. In this paper we used an adaptive neural fuzzy filter as an ECG beat classifier. We combined 3 famous wavelet transforms and used them mid 4 the order AR model coefficient as features. Our results suggest that a new proposed classifier (ANFF) with these features can generalize better than ordinary MLP architecture and also learn better and faster. The results of proposed method show high accuracy in ECG beat classification (97.6\%) with $100 \%$ specificity and high sensitivity.
\end{abstract}

Keywords: Adaptive Neural Fuzzy Filter, ECG Arrhythmia Classification, Pattern Recognition, Multilayer Perceptron.

\section{INTRODUCTION}

Electrocardiography deals with the electrical activity of the heart. Bio-signals being non-stationary signals, the reflection may occur at random in the time-scale. Therefore, for effective diagnostic, ECG pattern and heart rate variability may have to be observed over several hours. Thus the volume of the data being enormous, the study is tedious and time consuming. Therefore, computer-based analysis and classification of cardiac diseases can be very helpful in diagnostic [1]. Several algorithms have been developed in the literature for detection and classification of ECG beats. One of ECG beat recognition is neural network classification method [2-9]. Multilayer perceptron (MLP), has been shown to be able to recognize and classify ECG signals more accurately. However conventional Neural Networks with Back Propagation algorithm (BPNN) suffers from slow convergence to local and global minima and from random settings of initial of weights, which may make the neural networks have very poor mappings from inputs to output. More over in conventional neural networks, users have to determine the structure of network such as the numbers of hidden layer before training and it is too hard to select proper structure. Another ECG classification method is Fuzzy Hybrid Neural Network [10]. In this structure a FCM algorithm is used to clustering the features and the center of clusters will be used as the input of MLP neural network. This structure made the results conventional MLP better but because of using MLP as final classifier, the shortcomings of MLP are still exist.

To overcome the shortcomings encountered in neural networks, while still keeping their advantages, an adaptive neural fuzzy filter, (ANFF) has been developed in [1]. The ANFF is a feed forward multilayer network that integrates the basic elements and functions of a traditional fuzzy system into a connectionist structure. An important feature of this adaptive filter is that it can dynamically partition the input space and output space using irregular fuzzy hyper box [11]. For the adaptation of membership functions in the ANFF, the back propagation algorithm is used to find the optimal parameters under the mean square error (MSE) criterion. Hence, in the ANFF, the Fuzzy ART is used for structure learning and the back propagation algorithm for parameter learning. The ANFF can thus on-line partition the input-output spaces, tune membership functions, and find proper fuzzy logic rules dynamically on the fly. Users need not give the initial fuzzy partitions, membership functions, or fuzzy logic rules except for the case that 
expert knowledge is available and is used as the initial fuzzy rules. Hence, there are no hidden nodes in the beginning of learning; they are created and begin to grow as the training signal arrives. Since the structure of the ANFF is constructed from fuzzy if-then rules, once the input-output relationship is constructed, it will not be destroyed and, thus, no knowledge forgetting may happen while in conventional neural network we might had this event. These properties can make the ANFF more suitable for on-line classification than neural networks. Therefore in this paper we decided to use this filter as a classifier instead of a filter. More over, in this paper we used the fuzzy combination of 3 wavelets which were: Daubechies, Symlet, Biorthogonal mid $4^{\text {th }}$ order AR model coefficients as features. These features beside ANFF had more efficient results than MLP. This algorithm was faster and more reliable than MLP and it has high mean accuracy $97.6 \%$ and also high sensitivity and specificity.

This paper organized as follows. Section 1 describes the basic structure and functions of ANFF in brief. The on-line structure/parameter learning algorithm of the ANFF, which combines fuzzy ART and back propagation learning algorithm under the MSE criterion is presented in Section 3. In Section 4 we describe the feature extraction method. In Section 6 we will show the results of this method and compare this method with conventional neural network (MLP). Finally conclusions are summarized in the last section.

\section{THE STRUCTURE OF ADAPTIVE NEURAL FUZZY FILTER}

\subsection{Adaptive Neural Fuzzy Filters}

In this section, we will describe the structure and functions of ANFF briefly. The ANFF (see Figure 1) has five layers with node and link numbering defined by the brackets on the left-hand side of the figure.

Layer-1 nodes are input nodes representing input variables. Layer-5 nodes are output nodes representing output variables. Layer- 2 and layer- 4 nodes are term nodes that act as membership functions representing the terms of respective input and output variables. Each layer-3 node is a rule node representing one fuzzy logic rule. Thus, together all the layer- 3 nodes will be as a fuzzy rule base. The links between layers 3 and 4 function as a connectionist inference engine. Layer-3 links define the preconditions of rule nodes, and layer-4 links define the consequents of the rule nodes. Therefore, each rule node has at most one link to some term node of a linguistic node, and may have no such links. This is true both for precondition links (link in layer 3 ) and consequent links (links in layer 4). The links in layers 2 and 5 are fully connected between linguistic nodes and their corresponding term nodes. The arrows indicate the normal signal flow directions when the network is in operation (after it has been built and trained). When we are in structure learning step, ANFF operates in Up-Down mode and when we want to obtain estimated output,
ANFF operates in Down-Up mode.

The ANFF uses the technique of complement coding from fuzzy ART [12] to normalize the input-output training vectors. Complement coding is a normalization process that rescales an $\mathrm{n}$-dimensional vector, $\mathbf{x}=(\mathrm{x} 1$, $\mathrm{x} 2 \ldots \mathrm{xn}$ ), to its $2 \mathrm{n}$-dimensional complement coding such that

$$
\begin{aligned}
x^{\prime} & \equiv\left(\bar{x}_{1}, \bar{x}_{1}^{c}, \bar{x}_{2}, \bar{x}_{2}^{c}, \cdots, \bar{x}_{n}, \bar{x}_{n}^{c}\right) \\
& \equiv\left(\bar{x}_{1}, 1-\bar{x}_{1}, \bar{x}_{2}, 1,-\bar{x}_{2}, \cdots, \bar{x}_{n}, 1-\bar{x}_{n}\right)
\end{aligned}
$$

where $\quad x^{\prime} \equiv\left(\bar{x}_{1}, \bar{x}_{2}, \ldots \bar{x}_{n}\right)=\bar{x}=\frac{x}{\|x\|} \quad$ and $\quad \bar{x}_{i}^{c} \quad$ is the complement of $\bar{x}_{i}$, i.e., $\bar{x}_{i}^{c}=1-\bar{x}_{i}$. As mentioned in [11], complement coding helps avoid the problem of category proliferation when using fuzzy ART clustering. It also preserves training vector amplitude information. In applying the complement coding technique to the ANFF, all training vectors (either input state vectors or desired output vectors) are transformed to their complement coded form in the preprocessing process, and the transformed vectors are then used for training.

A typical network consists of nodes with some finite number of fan-in connectionist from other nodes represented by weight values, and fan-out connectionists to other nodes. Associated with the fan-in of a node is an integration function $f$ which combines information, activation, or evidence from other nodes, and provides the net input, i.e.,

$$
\text { net -input }=f\left(z_{1}^{(k)}, z_{2}^{(k)}, \ldots z_{p}^{(k)} ; w_{1}^{(k)}, w_{2}^{(k)}, \ldots w_{p}^{(k)}\right)
$$

where $Z_{i}^{(k)}(i), \ldots p$ is the ith input to a node in layer $\mathrm{k}$, and $w(i)$ is the weight of the associated link. The superscript in the above equation indicates the layer number. This notation will be also used in the following equations. Each node also outputs an activation value as a function of its net-input.

$$
\text { output }=a(f)
$$

The description of the functions of nodes in each of five layers of the ANFF can be obtained from Reference [1].

\subsection{The Structure-Learning Step}

The structure learning step consists of three learning processes: input fuzzy clustering process, output fuzzy clustering process, and mapping process. The first two processes are performed simultaneously on both sides of network, and are described below.

\subsubsection{Input Fuzzy Clustering Process}

For input fuzzy clustering, ANFF uses the fuzzy ART fast learning algorithm [11,12] to find the input membership function parameters $V_{i j}^{(2)}, U_{i j}^{(2)}$. For this purpose, first, the values of choice functions, $\mathrm{Tj}$, for each complement coded vector are computed by 


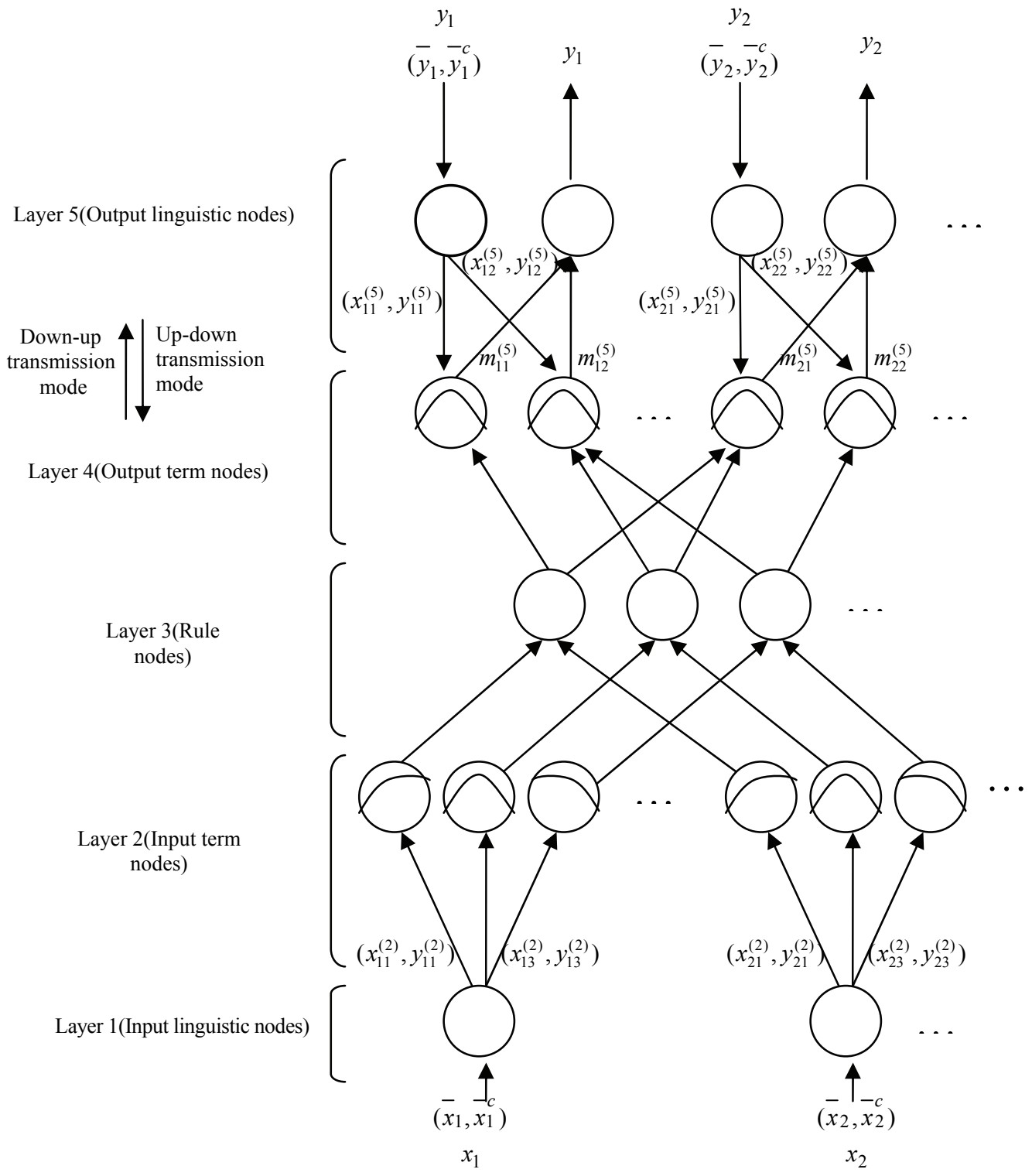

Figure 1. Structure of Adaptive Neural Fuzzy Filter (ANFF).

$$
T_{j}\left(x^{\prime}\right)=\frac{\left|x^{\prime} \wedge \omega_{j}\right|}{a+\left|\omega_{j}\right|} \quad j=1, \ldots ., N
$$

where " $\Lambda$ " is the minimum operator performed for the pairwise elements of two vectors, $\alpha \geq 0$ is a constant, $\mathrm{N}$ is the current number of rule nodes, and $\mathrm{wj}$ is the complement weight vector for each rule node.

Note that the choice function value indicates the similarity between the input vector $\mathbf{x}^{\prime}$ and the complement weight vector $\mathbf{w j}$. We then need to find the complement weight vector closest to $\mathbf{x}^{\prime}$. The chosen category is indexed by $\mathbf{J}$, where

$$
\frac{\left|x^{\prime} \wedge \omega_{j}\right|}{\left|x^{t}\right|} \geq \rho
$$

where $\rho$ is between 0 and 1 is a vigilance parameter. If the vigilance criterion is not met, we say mismatch reset occurs. In this case, the choice function value $\mathrm{TJ}$ is set to zero for the duration of the input presentation to prevent persistent selection of the same category during search (we call this action "disabling $\mathrm{J}$ "). A new index $\mathrm{J}$ is then chosen using (20). The search process continues until the chosen J satisfies (21). If no such $\mathrm{J}$ is found, then a new input hype box is created by adding a set of $\mathrm{n}$ new input term nodes, one for each input linguistic variable, and setting up links between the newly added input term nodes and the input linguistic nodes. The complement weight vectors on these new layer-2 links are simply given as the current input vector, $\mathrm{x}^{\prime}$. These newly added input term nodes and links define a new hyper box, and thus a new category, in the input space. We denote this newly added hyper box as J. 


\subsubsection{Output Fuzzy Clustering Process}

The output fuzzy clustering process is exactly the same as the input fuzzy clustering process except that it is performed between layers 4 \& 5 which are working in the up-down transmission mode.

\subsubsection{Mapping Process}

After the two hyper boxes in the input and output spaces are chosen in the input and output fuzzy clustering processes, the next step is to perform the mapping process which decides the connections between layer-3 and layer-z4 nodes. This mapping process is described by the following algorithm, wherein connecting rule node $\mathrm{J}$ output hyper box $\mathrm{K}$ we means connecting the rule node $\mathrm{J}$ to the output term nodes that constitutes the hyper box $\mathrm{K}$ in the output space.

Step 1: IF rule node $\mathrm{J}$ is a newly added node THEN connect rule node $\mathrm{J}$ to output hyper box K.

Step 2: ELSE IF rule node $\mathrm{J}$ not connected to output hyper box K originally THEN disable $\mathrm{J}$ and perform Input Fuzzy Clustering Process to find the next qualified J.

Step 3: ELSE no structure change is necessary. In the mapping process, hyper boxes $\mathrm{J}$ and $\mathrm{K}$ are resized according to the fast learning rule [40] by updating weights, $\mathrm{WJ}$ and $\mathrm{WK}$, as

$$
W_{J}^{(\text {new })}=x^{\prime} \wedge W_{J}^{(\text {old })} \quad, W_{k}^{(\text {new })}=x^{\prime} \wedge W_{k}^{(\text {old })}
$$

\subsection{The Parameter-Learning Step}

After the network structure has been adjusted according to the current training pattern in the structure-learning step, it is then necessary to fine tune the network parameters using the same training pattern. Basically, the back propagation algorithm is used to find node output errors, which are then analyzed to guide parameter adjustment. As mentioned above, the goal of training the ANFF is to minimize the error function

$$
E=\frac{1}{2}(S(K)-\hat{S}(K))^{2}
$$

where $s(k)$ is the desired signal, and $\mathrm{s}^{\wedge}(\mathrm{k})$ is the filtered signal. Based upon this MSE criterion and in analogy to the back propagation algorithm, we can derive the following

$$
\begin{aligned}
& W(K+1)=W(K)+\Delta W(K)=W(K)+\eta\left(-\frac{\partial E}{\partial w}\right) \\
& -\frac{\partial E}{\partial W}=-\frac{\partial E}{\partial F} \cdot \frac{\partial F}{\partial W}=-\frac{\partial E}{\partial \alpha} \cdot \frac{\partial \alpha}{\partial f} \cdot \frac{\partial f}{\partial w}
\end{aligned}
$$

where $\mathrm{W}$ is the adjustable parameter in the filter.

The adapting equations of the parameters of each layer can be obtained from [1] in more details.

\section{DATA ACQUISITION AND FEATURE EXTRACTION METHOD}

We had 4 kinds of ECG beats. There were: Atrial Fibrillation beats (AF), Ventricular Tachycardia beats (VT),
Super Ventricular Tachycardia Arrhythmia beats (SVA) and Normal beats. We selected 18 signals from each kind of beat from MIT-BIH Arrhythmia database. So we had 72 signals totally. The arrhythmia database of MIT-BIH, have been preprocessed in such away that they are clean from any corrupting noise. Therefore we did not need to do any additional preprocessing on our ECG signals. The sampling frequency of MIT-BIH Arrhythmia database is $250 \mathrm{~Hz}$. We used a 1000 point moving window with 200 point overlap between each two windows for each ECG signal. So each ECG signal converted to 24 segments which each segment had 1000 point. Therefore we had 1728 segment totally. We used $80 \%$ of these segments for training ANFF and $20 \%$ of that to test the performance of ANFF.

We used a fuzzy combination of 3 wavelet transform which were: Daubechies (db4), Symlets (sym8) and Biorthoginal (bior4.4). A 4 level decomposition was done with each wavelet transform. And then we selected the maximum value between these 3 wavelets in each level. Therefore, 4 wavelet coefficients were obtained for each segment with this fuzzy combination if wavelet transforms. We also used the 4th order AR model coefficients as another feature extraction method so 4 features were obtained for each segment by this method. Therefore finally we had an 8 dimensional feature vector for each segment. Then we used these features as input for ANFF. We also defined the target for each segment and used them as output variable for ANFF.

\section{RESULTS}

We considered following values for the constants in ANFF: learning parameter $(\eta)=0.1$, fuzziness parameter $(\gamma)=0.6$, choice function parameter $(\alpha)=0.7$.

In the first step of process we used only the Daubechies wavelet coefficients as ECG features. In the second step of process we used Daubechies wavelet mid 4th order AR model coefficient as ECG features. In third step of process we used only the fuzzy combination of three wavelets as ECG features. And finally we used fuzzy combination of wavelets mid 4th order AR model coefficient as ECG features. Table 1 shows the results of using these features with ANFF separately. Then also did these steps with an MLP with 40 hidden nodes. Table 2 shows the results of using these features with MLP. In both methods (ANFF and MLP) we calculated specificity and sensitivity and accuracy for each kind of features. We selected 1382 segments for training and 346 segments for test randomly.

As we can see from Table 1 and Table 2, among 4 kinds of features extracted, the fuzzy combination of 3 pre nominate wavelet transforms mid 4th order AR model coefficients provided best results for both ANFF and MLP structures. Also we can see that in compare with MLP, ANFF had much better results. ANFF had high accuracy about $97.6 \%$ and also it had higher specificity and sensitivity than MLP. Furthermore unlike the MLP, ANFF did not need to have pre determined struc- 
Table 1. Results of ANFF in ECG beat recognition.

\begin{tabular}{lcccc}
\hline \multicolumn{1}{c}{ Classifier } & Total test segments & SE(\%) & SP(\%) & Accuracy(\%) \\
\hline $\begin{array}{l}\text { ANFF with wavelet features } \\
\text { ANFF with wavelet and AR model } \\
\text { coefficients features }\end{array}$ & 346 & $92.7 \%$ & $98.1 \%$ & $90.33 \%$ \\
$\begin{array}{l}\text { ANFF with fuzzy combination of } \\
\text { wavelets }\end{array}$ & 346 & $95.7 \%$ & $98.4 \%$ & $94.2 \%$ \\
$\begin{array}{l}\text { ANFF with fuzzy combination of } \\
\text { wavelets and AR model coefficient } \\
\text { and features }\end{array}$ & 346 & $96.6 \%$ & $98.6 \%$ & $92.1 \%$ \\
\hline
\end{tabular}

Table 2. Results multi layer perceptron meural network (MLP) in ECG beat recognition.

\begin{tabular}{lcccc}
\multicolumn{1}{c}{ Classifier } & Total test segments & SE(\%) & SP(\%) & Accuracy(\%) \\
\hline $\begin{array}{l}\text { MLP with wavelet features } \\
\text { MLP with wavelet and AR model }\end{array}$ & 346 & $91.4 \%$ & $96.7 \%$ & $86.7 \%$ \\
coefficients features & 346 & $93.49 \%$ & $96.93 \%$ & $91.3 \%$ \\
$\begin{array}{l}\text { MLP with fuzzy combination of } \\
\text { wavelets }\end{array}$ & 346 & $95.3 \%$ & $97.47 \%$ & $88.7 \%$ \\
$\begin{array}{l}\text { MLP with fuzzy combination of } \\
\text { wavelets and AR model coefficient } \\
\text { features }\end{array}$ & 346 & $94.76 \%$ & $99.6 \%$ & $94.2 \%$ \\
\hline
\end{tabular}

ture and it will find the best structure during training by itself.

\section{CONCLUSION}

In this paper an ANFF has been developed to classify ECG signals by using 4 different features set. Results show that among these features set, a fuzzy combination of Daubeches, Symlets and Biorthogonal wavelet transforms mid $4^{\text {th }}$ order AR model coefficients, had best results.

As we know, several algorithms have been developed for ECG beat recognition in literatures. Most of these algorithms have used Neural Networks as their final classifier. There are several kinds of Neural Networks. One of the most useful neural networks in ECG classification is Multi Layer Perceptron (MLP) neural network. It is easy to use and it has been shown reliable results in ECG beat classification. Therefore we decided to compare the performance of our purposed method with MLPNeural Networks, such as MLP, are capable in classification. They have strong learning and generalization ability but they have some disadvantages. For example: (1) we need iterative training cycles to encode the relation between inputs and outputs into the neural networks, so neural networks have long training time. (2) users have to predetermine the size and structure of neural networks (such as the numbers of nodes of hidden layers) initially and it is hard for users. (3) we can not use linguistic rules in neural networks directly.

Adaptive Neural Fuzzy Filters (ANFF) overcomes these shortcomings. In ANFF, users do not need to predetermine the numbers of nodes in hidden layer and these nodes are generated automatically during the training process. Since the structure of ANFF is optimum, it takes much shorter to train in compare with neural networks. More over, we can use linguistic rules, which are obtained from expert knowledge, in ANFF directly. ANFF was introduced by Chin-Teng Lin and Chia-Feng Juang in 1997 [1]. They introduced the structure of ANFF in details and they used ANFF as an adaptive filter for noise cancellation. We decided to use this useful filter as a classifier. Therefore in this paper we used ANFF as an ECG beat classifier and we compared its performance with most commonly used classifier (MLP).

On the other hand, wavelet transforms (WT) are must commonly feature extraction method in ECG beat recognition algorithms. An over view on previous works in this context shows that AR model beside wavelet transform has showed better results in ECG beat classification. More over, there are several mother wavelets which are used for ECG feature extraction. Therefore we decided to combine three mother wavelets which are most common used for ECG feature extraction and we used AR model coefficients beside this combination as our final feature extraction method.

A comparative assessment of performance of ANFF with MLP neural networks show that more reliable results are obtained with the ANFF for the classification of ECG signals.

MLP neural networks are still able to generalize with good recognition accuracy. However, they take longer to train and users have to predetermine the size and structure of network such as the number of hidden layers and it is truly difficult. The aim in developing ANFF was to achieve more optimum results with relatively few signal features. It has been demonstrated that the training time of ANFF was much shorter than time required by MLP and the accuracy (97.6\%), specificity $(100 \%)$ and sensitivity $(97.6 \%)$ of ANFF were much 
better than those of MLP. Furthermore the ANFF can be trained by numerical data and linguistic information expressed by fuzzy if-then rules. Another key feature of ANFF is that, without any given initial structure, the ANFF can construct itself automatically from numerical training data.

The proposed method, which incorporates the techniques of Adaptive Neural Fuzzy Filter and back propagation learning and combines their advantages, can be said to be more capable of recognizing other biological signals than conventional Neural Networks with Back Propagation algorithm (BPNN) such as Multi Layer Perceptron (MLP).

\section{REFERENCES}

[1] C.T. Lin, C.F. Juang, (2001) "An adaptive neural fuzzy filter and its applications," IEEE Transactions On Systems, MAN, And Cybernetics, VOL. 27, NO. 4, 1103-1110.

[2] S. Osowaki, T.H. Linh, (2001) "ECG beat recognition using fuzzy hybrid neural network," IEEE Trans. Biomed. Eng. 48 (11) 1265-1271.

[3] Y. Ozbay, B. Karlik, (2001) "A reconition of ECG arrhythmias using artificial neyral network," Proceedings of the $23^{\text {rd }}$ Annual Conference, IEEE/EMBS, Istanbul, Turkey, pp. 76-80.

[4] Y. Ozbay, "Fast recognition of ECG arrhythmias," (1999) Ph.D. Yhesis, Institute of Natural and Applied Science, Selcuk Univer- sity,

[5] S.Y. Foo, G. Harvey, A. Meyer-Baese, (2002) "Neural networkbased ECG pattern recognition", Eng. Appl. Artif. Intell. 15, 353-360.

[6] V. Pilla, H.S. Lopes, (1999) "Evolutionary training of a neuro-fuzzy network for detection of a P wave of the ECG," Proceeding of the third international conference on computational intelligence and multimedia applications, New Dehli, India, 102-106.

[7] M. Engin, S. Demirag, (2003) "Fuzzy-hybrid neural network based ECG beat recognition using three different types of feature sets," Cardiovasc. Eng. Int. J. 3 (2) 71-80.

[8] S. Hykin, (1994) Neural Networks: A comperhensive Foundation, Macmillan, New York.

[9] B. Karlik, m.o. Tokhi, M. Alci, (2003) "A fuzzy clustering neural network architecture for multifunction upper-limb prosthesis," IEEE Trans. Biomed. Eng. 50 (11), 1255-1261.

[10] R. Acharya, P.S. Bhat, S.S. Iyengar, A. Roo, S. Dua, (2001) "Classification of heart rate data using artificial neural network and fuzzy equivalence relation," J. Pattern Recognition Soc, 4, 238-244.

[11] G. A. Carpenter, S. Grossberg, and D. B. Rosen, (2001) "Fuzzy ART: Fast stable learning and categorization of analog patterns by an adaptive adaptive resonance system," Neural Networks, 4, 759-771.

[12] G. A. Carpenter, S. Grossberg, N. Markuzon, J. H. Reynolds, and D. B. Rosen, (2002) "Fuzzy ARTMAP: A neural network architecture for incremental supervised learning of analog multidimensional maps," IEEE Trans. Neural Networks, vol. 3, pp. 698-712. 